\title{
Electron Holography of the Magnetic Phase Shift of a Current-Carrying Wire
}

Amir H. Tavabi ${ }^{1}$, Vadim Migunov ${ }^{1}$, Alexey Savenko ${ }^{2}$ and Rafal E. Dunin-Borkowski ${ }^{1}$

${ }^{1}$ Ernst Ruska-Centre for Microscopy and Spectroscopy with Electrons and Peter Grünberg Institute, Forschungszentrum Jülich, D-52428 Jülich, Germany

${ }^{2}$ Central Institute for Engineering, Electronics and Analytics, Forschungszentrum Jülich, D-52428 Jülich, Germany

The controlled generation of magnetic fields to study switching processes in magnetic materials in the transmission electron microscope (TEM) is highly challenging [1-3]. Here, we use electron holography to study the magnetic field around a carefully fabricated current-carrying wire. As a result of the fact that a current flowing parallel to the plane of an untilted TEM specimen produces no net magnetic phase shift, we examine a short nano-fabricated segment of wire that is oriented parallel to the electron beam direction. Different regions of such a wire could in principle be used to apply either in-plane or out-of-plane magnetic fields to closely-adjacent nanomagnets.

A free-standing three dimensional nanoscale circuit was created by using focused ion beam (FIB) milling to sculpt electrodes in the forms of a hook and a needle from a chemically-etched 250- $\mu \mathrm{m}$-diameter $\mathrm{Au}$ wire. FIB milling was carried out in an FEI Helios NanoLab 600i dual-beam workstation using $30 \mathrm{kV}$ Ga ions and ion beam currents of $21 \mathrm{nA}$ to $7.7 \mathrm{pA}$. The hook and needle were mounted in a NanoFactory scanning tunneling microscopy specimen holder, as shown in Fig. 1. The piezo-driven tip in the holder was used to move the needle towards the hook electrode to create an electrical contact. Contact between the needle and the hook was initially made with the specimen tilted to an angle of $56^{\circ}$, before returning the specimen tilt angle to $0^{\circ}$, with the arm of the hook oriented parallel to the electron beam direction, to perform measurements using electron holography. The current-voltage characteristic of the circuit was measured to be linear and unaffected by electron beam irradiation in the TEM.

Off-axis electron holograms of the magnetic field around the vertical segment of the wire were recorded at $300 \mathrm{kV}$ in an FEI Titan 60-300 TEM equipped with an XFEG field emission gun and two electron biprisms. Observations were performed in Lorentz mode in a magnetic-field-free environment (with the conventional microscope objective lens switched off).

Figures 2 a) and b) show 6-times-amplified phase contour maps recorded for currents through the wire of 105 and $500 \mu \mathrm{A}$, respectively. The contours originate from the presence of an azimuthal magnetic field around the short section of wire that is oriented parallel to the electron beam direction. Line profiles measured from the phase images as a function of distance from the short section of wire are shown in Figs. 2 c) and d), respectively. The gradient of the measured phase profiles increased with the magnitude of the current in the circuit, but was not identical when the direction of the current was reversed, probably due to the build-up of electron-beam-induced charge in the region of interest.

\section{References:}

[1] G. Yi et al., Ultramicroscopy 99 (2004) p. 65-72.

[2] T. Uhlig, M. Heumann, J. Zweck, Ultramicroscopy 94 (2003) p. 193-196. 
[3] K. Yamamoto et al., Ultramicroscopy 106 (2006) p. 314-319.

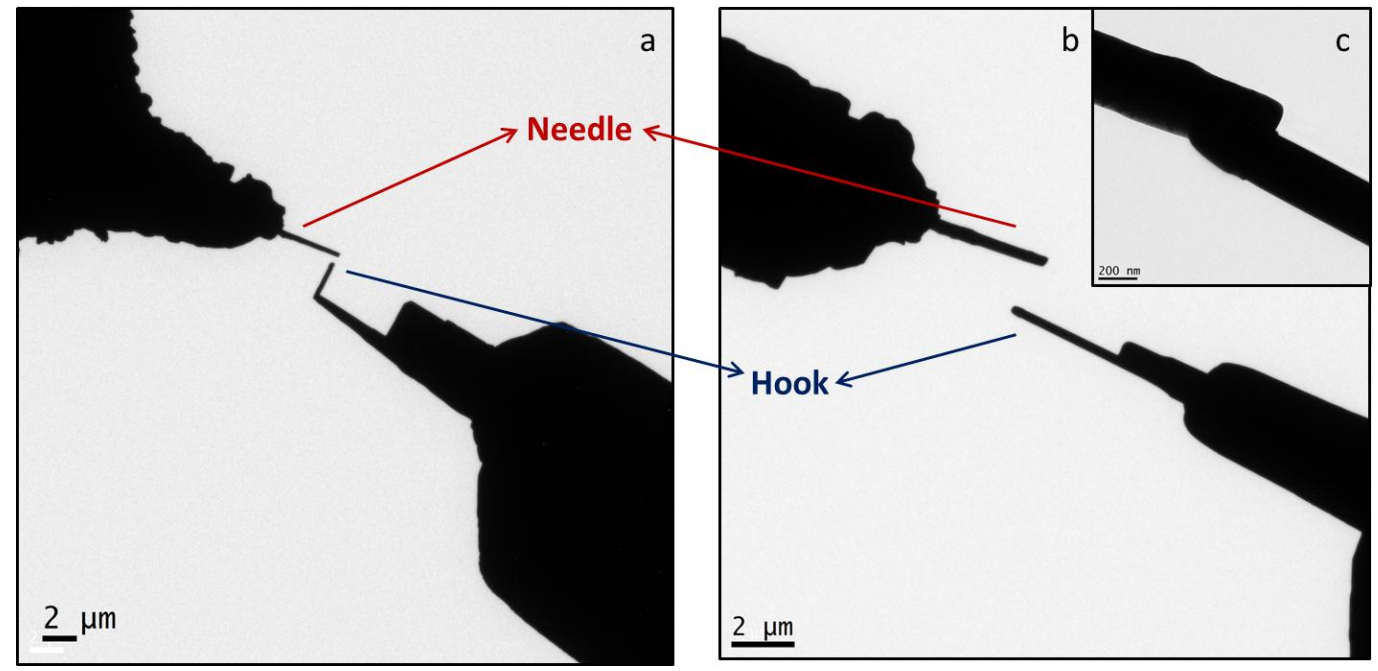

Figure 1. Bright-field TEM images of FIB-milled hook and needle electrodes with the specimen tilted by a) 56 and b) $0^{\circ}$; c) BF image recorded with the circuit closed to allow current to flow and with the arm of the hook oriented parallel to the electron beam direction.
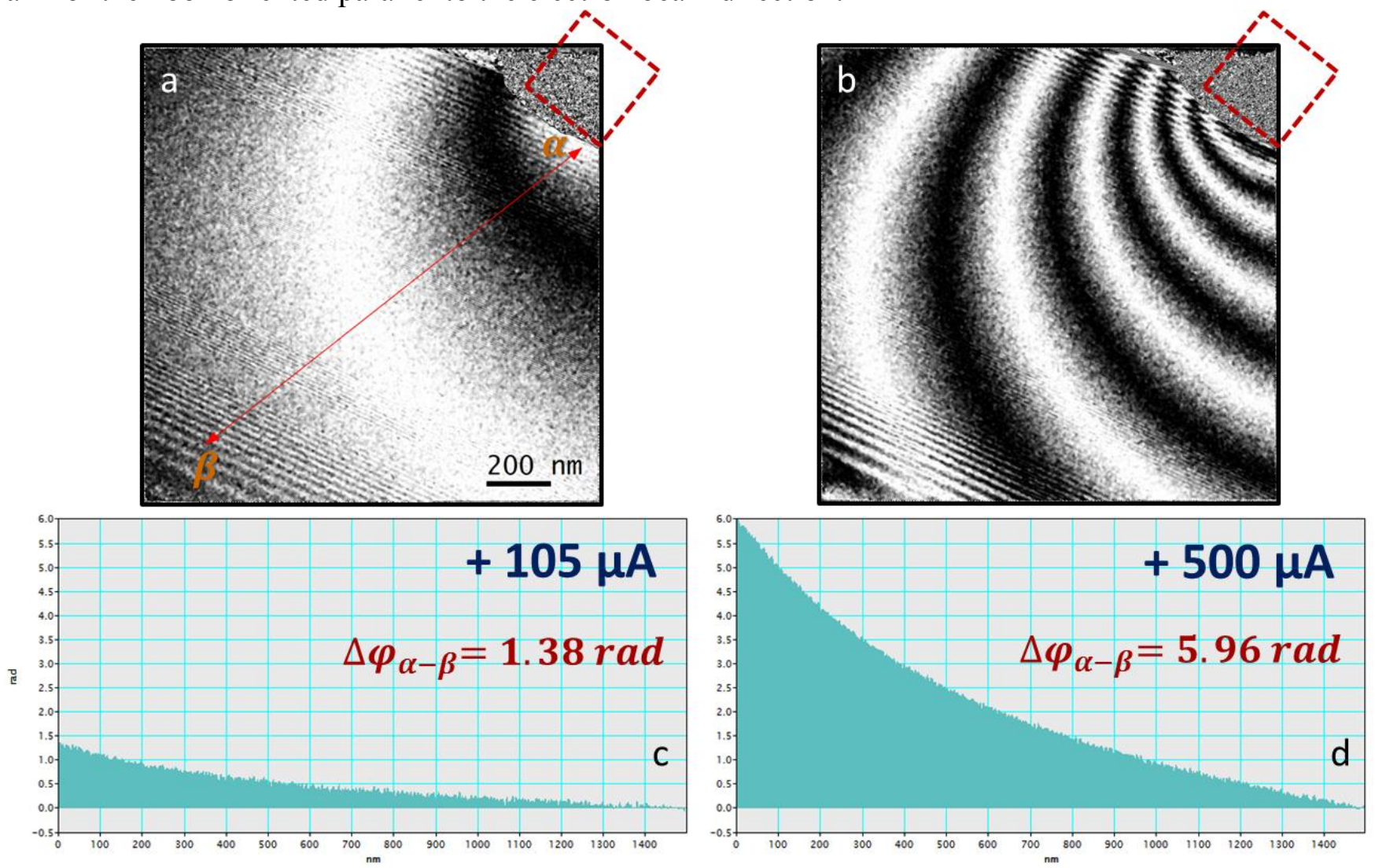

Figure 2. a), b) 6-times-amplified phase images illustrating the development of an in-plane magnetic field around the short segment of the current-carrying wire, recorded for currents of a) 105 and b) 500 $\mu \mathrm{A}$. The red dashed square shows the position of the arm of the hook. c) and d) show phase profiles extracted from a) and b), respectively, along the line from point $\alpha$ to point $\beta$ indicated in a). Each line profile is averaged over a distance of $40 \mathrm{~nm}$ in the original phase image. 